\title{
Approximations of some near open sets in ideal topological spaces
}

\author{
A. S. Nawar
}

Correspondence:

ashraf_nawar2020@yahoo.com Department of Mathematics and

Computer Science, Faculty of Science, Menoufia University,

Menoufia, Egypt

\begin{abstract}
In this paper, we introduce the concepts of $j$-ideal lower and $j$-ideal upper approximations as a generalization of approximation by Pawlak by using $j$-ideal open sets where $j \in\left\{a, S, P, \gamma, \beta, \wedge_{\beta}\right\}$. Some of their basic properties with the aid of examples are proven. Furthermore, we study the relationships between these approximations to get the best of them.
\end{abstract}

Keywords: $j$-ideal approximation spaces, $j$-ideal open sets, Accuracy measure

\section{Introduction}

Rough set theory was originally proposed by Pawlak [1,2] for dealing with uncertain knowledge in information systems. Using the concepts of lower and upper approximation in rough set theory, knowledge hidden in information systems may be unraveled and expressed in the form of decision rules. So far, rough set theory has been successfully applied in fields such as machine learning and knowledge discovery [3, 4], data mining [5, 6], decision-making support and analysis [7-9], process control [10, 11], expert system [12], and pattern recognition [13]. Kuratowski [14] and Vaidyanathaswamy [15] introduced and investigated the concept of ideals in topological spaces. In 1990, Jankovic and Hamlett [16] investigated further properties of ideal topological spaces. In this paper, the notions of $j$-ideal lower and $j$-ideal upper approximations where $j \in\left\{\alpha, S, P, \gamma, \beta, \wedge_{\beta}\right\}$ as a generalization of approximation by Pawlak via $j$-ideal open sets are introduced and studied. Some of their basic properties with the aid of examples are investigated. Moreover, the relationships between these approximations are presented.

\section{Preliminaries}

Throughout this paper, $(X, \tau)$ (or simply $X$ ) represent topological spaces on which no separation axioms are assumed unless otherwise mentioned. For a subset $A$ of $X, \operatorname{cl}(A)$, $\operatorname{int}(A)$, and $A^{c}$ denote the closure of $A$, the interior of $A$ and the complement of $A$, respectively. Let us recall the following definitions, which are useful in the sequel.

Definition 2.1 A subset $A$ of a topological space $(X, \tau)$ is called:

(1) Preopen [17] if $A \subseteq \operatorname{int}(\operatorname{cl}(A))$ and preclosed if $\operatorname{cl}(\operatorname{int}(A)) \subseteq A$.

(2) Semi-open [18] if $A \subseteq \operatorname{cl}(\operatorname{int}(A))$ and semi-closed if $\operatorname{int}(\operatorname{cl}(A)) \subseteq A$.

(3) b-open [19] $\gamma$-open [20] if $A \subseteq \operatorname{cl}(\operatorname{int}(A)) \cup \operatorname{int}(\operatorname{cl}(A))$. 
(4) Semi-preopen [21] (= $\beta$-open [22]) if $A \subseteq \operatorname{cl}(\operatorname{int}(\operatorname{cl}(A)))$ and semi-preclosed $(=\beta$-closed $)$ if $\operatorname{int}(\operatorname{cl}(\operatorname{int}(A))) \subseteq A$.

(5) $\alpha$-open [23] if $A \subseteq \operatorname{int}(\operatorname{cl}(\operatorname{int}(A)))$ and $\alpha$-closed if $\operatorname{cl}(\operatorname{int}(\operatorname{cl}(A))) \subseteq A$.

The class of all preopen (resp. semi-open, $\gamma$-open, semi-preopen, and $\alpha$-open) sets in a topological space $(X, \tau)$ is denoted by $P O(X)$ (resp. $S O(X), \gamma O(X), S P O(X)$, and $\alpha O(X)$ ). All of these classes are larger than $\tau$ and closed under arbitrary unions. The class of all preclosed (resp. semi-closed, $\gamma$-closed, semi-preclosed, and $\alpha$-closed) sets in a topological space $(X, \tau)$ is denoted by $P C(X)$ (resp. $S O(X), \gamma C(X), S P C(X)$, and $\alpha C(X)$ ).

Definition 2.2 Let $A$ be a subset of a topological space $(X, \tau)$. A subset $\wedge_{\beta}(A)$ is defined as follows [24]: $\wedge_{\beta}(A)=\cap\{G: A \subseteq G, G \in \beta O(X)\}$. The complement of $\wedge_{\beta}(A)$-set is called $\vee_{\beta}(A)$-set.

Definition 2.3 Let $(X, \tau)$ be a topological space and $A \subseteq X$. A subset $A$ is called $\wedge_{\beta}$-set [24] if $A=\wedge_{\beta}(A)$. The family of all $\wedge_{\beta}$-set and $\vee_{\beta}$-set are denoted by $\wedge_{\beta} O(X)$ and $\vee_{\beta} C(X)$, respectively.

Definition 2.4 [16] An ideal $I$ on a topological space $(X, \tau)$ is a non-empty collection of subsets of $X$ which satisfies the following two conditions:

(i) If $A \in I$ and $B \subseteq A$, then $B \in I$ (heredity).

(ii) If $A \in I$ and $B \in I$, then $A \quad B \in I$ (finite additivity).

An ideal topological space $(X, \tau)$ with an ideal $I$ on $X$ is denoted by $(X, \tau, I)$.

Definition 2.5 For a subset $A \subseteq X, A^{*}(I, \tau)=\{x \in X: G \cap A \notin I$ for each neighborhood $G$ of $x\}$ is called the local function of $A$ with respect to $I$ and $\tau$ [14]. We simply write $A^{*}$ instead of $A^{*}(I, \tau)$ in case there is no chance for confusion.

For every ideal topological space $(X, \tau, I)$, there exists a topology $\tau^{\prime \prime}(I)$, finer than $\tau$, generated by the base $\beta(I, \tau)=\{G-i: G \in \tau, i \in I\}[16]$. Additionally, $\operatorname{cl}^{*}(A)=A \cup A^{*}$ defines a Kuratowski closure operator for $\tau^{\prime \prime}(I)$.

Definition 2.6 A subset $A$ of an ideal topological space $(X, \tau, I)$ is said to be:

(i) pre-I-open [25] if $A \subseteq \operatorname{int}\left(\mathrm{cl}^{\prime \prime}(A)\right)$.

(ii) semi- $I$-open [26] if $A \subseteq \mathrm{cl}^{*}(\operatorname{int}(A))$.

(iii) $\alpha-I$-open [26] if $A \subseteq \operatorname{int}\left(\mathrm{cl}^{*}(\operatorname{int}(A))\right)$.

(iv) $\gamma$-I-open [27] if $A \subseteq \mathrm{cl}^{*}(\operatorname{int}(A)) \cup \operatorname{int}\left(\mathrm{cl}^{*}(A)\right)$.

(v) $\beta$-I-open [26] if $A \subseteq \mathrm{cl}\left(\operatorname{int}\left(\mathrm{cl}^{*}(A)\right)\right)$.

Motivation for rough set theory has come from the need to represent subsets of a universe in terms of equivalence classes of a partition of that universe. The partition characterizing a topological space is called approximation space, where the set $X$ is called the universe and $R$ is an equivalence relation [28, 29]. The equivalence classes of $R$ are also known as the granules or elementary sets or blocks. We shall use $R_{x}$ to denote the equivalence class containing $x \in X$ and $X / R$ to denote the set of all elementary sets of $R$. In the approximation space $K=(X, R)$, the lower (resp. upper) approximation of a subset $A$ of $X$ is given by

$$
\underline{R}(A)=\left\{x \in X: R_{x} \subseteq A\right\} \text { (resp. } \bar{R}(A)=\left\{x \in X: R_{x} \cap A \neq \varnothing\right\} \text { where } R_{x}=\{x \in X: x R y\}
$$

Pawlak noted [30] that the approximation space with equivalence relation $R$ defines a uniquely topological space $(X, \tau)$ where $\tau$ is the family of all clopen sets in $(X, \tau)$ and $X /$ $R$ is a base of $\tau$. Moreover, the lower (resp. upper) approximation of any subset $A$ of $X$ is exactly the interior (resp. closure) of $A$. 
If $R$ is a general binary relation, then the approximation space $K=(X, R)$ defines a uniquely topological space $\left(X, \tau_{K}\right)$, where $\tau_{K}$ is the topology associated to $K$ (i.e., $\tau_{K}$ is the family of all open sets in $\left(X, \tau_{K}\right)$ and $\mathcal{S}=\{x R: x \in X\}$ is a subbase of $\tau_{K}$, where $x R=\{$ $y \in X: x R y\})[31,32]$.

Definition 2.7 [31] Let $K=(X, R)$ be an approximation space with general binary relation $R$ and $\tau_{K}$ be a topology associated to $K$. The triple $\kappa=\left(X, R, \tau_{K}\right)$ is called a topologized approximation space.

Definition 2.8 [31] Let $\kappa=\left(X, R, \tau_{K}\right)$ be a topologized approximation space and $A \subseteq$ $X$. The lower, upper approximations, boundary, positive and negative regions, and accuracy of the approximation of $A$ are defined respectively as follows:

$$
\begin{aligned}
& \underline{R}_{\tau_{K}}(A)=\cup\left\{G \in \tau_{K}: G \subseteq A\right\}=\operatorname{int}(A), \text { where } \operatorname{int}(A) \text { represents the interior of } A . \\
& \bar{R}_{\tau_{K}}(A)=\cap\left\{F \in \tau_{K} c: A \subseteq F\right\}=\operatorname{cl}(A) \text {, where } \mathrm{cl}(A) \text { represents the closure of } A . \\
& B_{\tau_{K}}(A)=\bar{R}_{\tau_{K}}(A)-\underline{R}_{\tau_{K}}(A) . \\
& \operatorname{POS}_{\tau_{K}}(A)=\underline{R}_{\tau_{K}}(A) . \\
& \mathrm{NEG}_{\tau_{K}}(A)=X-\bar{R}_{\tau_{K}}(A) . \\
& \eta_{\tau_{K}}(A)=\left|\frac{\underline{R}_{\tau_{K}}(A)}{\bar{R}_{\tau_{K}}(A)}\right| \text {, where }\left|\bar{R}_{\tau_{K}}(A)\right| \neq 0 .
\end{aligned}
$$

Definition 2.9 [33] Let $\kappa=\left(X, R, \tau_{K}\right)$ be a topologized approximation space and $A \subseteq$ $X$. The $\wedge_{\beta}$-lower, $\wedge_{\beta}$-upper approximations, $\wedge_{\beta}$-boundary, $\wedge_{\beta}$-positive and $\wedge_{\beta}$-negative regions, and $\wedge_{\beta}$-accuracy of the approximation of $A$ are defined respectively by:

$$
\begin{aligned}
& \underline{R}_{\wedge_{\beta}}(A)=\cup\left\{G \in \wedge_{\beta} O(X): G \subseteq A\right\}, \bar{R}_{\wedge_{\beta}}(A)=\cap\left\{F \in \wedge_{\beta} C(X): A \subseteq F\right\} \\
& B_{\wedge_{\beta}}(A)=\bar{R}_{\wedge_{\beta}}(A)-\underline{R}_{\wedge_{\beta}}(A) . \\
& \operatorname{POS}_{\wedge_{\beta}}(A)=\underline{R}_{\wedge_{\beta}}(A) . \\
& \operatorname{NEG}_{\wedge_{\beta}}(A)=X-\bar{R}_{\wedge_{\beta}}(A) . \\
& \eta_{\wedge_{\beta}}(A)=\left|\frac{\underline{R}_{\Lambda_{\beta}}(A)}{\bar{R}_{\Lambda_{\beta}}(A)}\right|, \text { where }\left|\bar{R}_{\wedge_{\beta}}(A)\right| \neq 0 .
\end{aligned}
$$

\section{$\wedge_{\boldsymbol{\beta}} \mathrm{l}$-open sets and its properties}

In this section, the collection of $\wedge_{\beta} \mathrm{I}$-open sets in ideal topological spaces is introduced. The relationships and characterizations of the collection of $\wedge_{\beta} \mathrm{I}$-open sets are discussed.

Definition 3.1 Let $A$ be a subset of an ideal topological space $(X, \tau, I)$. A subset $\wedge_{\beta} I(A)$ is defined as follows: $\wedge_{\beta} I(A)=\cap\{G: A \subseteq G, G \in \beta I O(X)\}$. The complement of $\wedge_{\beta} I(A)$-set is called $\vee_{\beta} I(A)$-set.

Definition 3.2 Let $(X, \tau, I)$ be an ideal topological space and $A \subseteq X$. A subset $A$ is called $\wedge_{\beta} I$-set if $A=\wedge_{\beta} I(A)$. The family of all $\wedge_{\beta} I$-sets and $\vee_{\beta} I$-sets is denoted by $\wedge_{\beta} I O(X)$ and $\vee_{\beta} I C(X)$.

Some of fundamental properties of $\wedge_{\beta} I$-sets will be shown in the next proposition.

Proposition 3.1 If $A, B$, and $A_{\alpha}(\alpha \in \Gamma)$ are subsets of an ideal topological space $(X, \tau, I)$, then the following properties hold:

(i) $A \subseteq \wedge_{\beta} I(A) \subseteq \wedge_{\beta}(A)$

(ii) $\wedge_{\beta} I(\varnothing)=\varnothing$ and $\wedge_{\beta} I(X)=X$ 
(iii) If $A \subseteq B$, then $\wedge_{\beta} I(A) \subseteq \wedge_{\beta} I(B)$

(iv) $\wedge_{\beta} I\left(\wedge_{\beta} I(A)\right)=\wedge_{\beta} I(A)$

(v) If $A \in \beta I O(X)$, then $A=\wedge_{\beta} I(B)$

(vi) $\wedge_{\beta} I\left(\cup_{\alpha \in \Gamma} \mathrm{A}_{\alpha}\right)=\cup_{\alpha \in \Gamma} \wedge_{\beta} I\left(\mathrm{~A}_{\alpha}\right)$

$($ vii $) \wedge_{\beta} I\left(\underset{\alpha \in \Gamma}{\cap} \mathrm{A}_{\alpha}\right) \subseteq \underset{\alpha \in \Gamma}{\cap} \wedge_{\beta} I\left(\mathrm{~A}_{\alpha}\right)$

Proof: We proof only (vi) and the rest of the proof follows directly from Definition 3.1.

(viii) Suppose $x \notin \underset{\alpha \in \Gamma}{\cup} \wedge_{\beta} I\left(A_{\alpha}\right)$, then $x \notin \cup_{\alpha \in \Gamma} \wedge_{\beta} I\left(A_{\alpha}\right)$ for each $\alpha \in \Gamma$.

Therefore, for each $\alpha \in \Gamma$, there exists $G_{\alpha} \in \beta I O(X)$ such that $x \notin G_{\alpha}$ and $A_{\alpha} \subseteq G_{\alpha}$. Thus, $\cup_{\alpha \in \Gamma}\left(A_{\alpha}\right) \subseteq \cup_{\alpha \in \Gamma}\left(G_{\alpha}\right)$ and $\cup_{\alpha \in \Gamma}\left(G_{\alpha}\right) \in \beta I O(X)$ which does not contain $x$. This implies that $x \notin \wedge_{\beta}$ $I\left(\cup_{\alpha \in \Gamma} A_{\alpha}\right)$. Consequently, $\wedge_{\beta} I\left(\cup_{\alpha \in \Gamma} A_{\alpha}\right) \subseteq \cup_{\alpha \in \Gamma} \wedge_{\beta} I\left(A_{\alpha}\right)$. Obviously, $\underset{\alpha \in \Gamma}{\cup} \wedge_{\beta} I\left(A_{\alpha}\right) \subseteq \wedge_{\beta} I\left(\cup_{\alpha \in \Gamma} A_{\alpha}\right)$.

Hence,

$$
\wedge_{\beta} I\left(\cup_{\alpha \in \Gamma} A_{\alpha}\right)=\cup_{\alpha \in \Gamma} \wedge_{\beta} I\left(A_{\alpha}\right) .
$$

Remark 3.1 In (vii) of Proposition 3.1, the equality does not necessarily hold as shown by the following example.

Example 3.1 Let $U=\{a, b, c, d, e\}$ be a universe, $I=\{\varnothing,\{a\},\{c\},\{a, c\}\}$,

and

$R=\{(a, a),(a, e),(b, c),(b, d),(c, e),(d, a),(d, e),(e, e)\}$ be a binary relation on $U$. If $A=\{c\}$ and $B=\{d\}$, then $\wedge_{\beta} I(A)=\{c, d\}, \wedge_{\beta} I(B)=\{d\}$, and $\wedge_{\beta} I(A \cap B)=\varnothing$.

Proposition 3.2 Let $(X, \tau, I)$ be an ideal topological space. Then, the following statements hold:

(i) $\varnothing, X$ are $\wedge_{\beta} I$-sets.

(ii) $\wedge_{\beta} I(A)$ is $\wedge_{\beta} I$-set, for any set $A$ of $X$.

(iii) Every $\beta$-I- open is $\wedge_{\beta} I$-set.

(iv) Union of $\wedge_{\beta} I$-sets is $\wedge_{\beta} I$-set.

(v) Intersection of $\wedge_{\beta} I$-sets is $\wedge_{\beta} I$-set.

Proof. Follows directly from Proposition 3.1 and Definition 3.2.

Proposition 3.3 Let $(X, \tau, I)$ be an ideal topological space and $A$ be $\wedge_{\beta} I$-set where $A \subseteq B \subseteq \wedge_{\beta} I(A)$. Then, $B$ is $\wedge_{\beta} I$-set.

Proof. Let $A$ be $\wedge_{\beta} I$-set. Then, $\wedge_{\beta} I(A)=A$ and since $A \subseteq B \subseteq \wedge_{\beta} I(A)$. Hence, $B=\wedge_{\beta} I(A)$ and so $\wedge_{\beta} I(B)=\wedge_{\beta} I\left(\wedge_{\beta} I(A)\right)=\wedge_{\beta} I(A)=B$.

Remark 3.2 In an ideal topological space $(X, \tau, I)$, the following implications hold

Fig. 1.

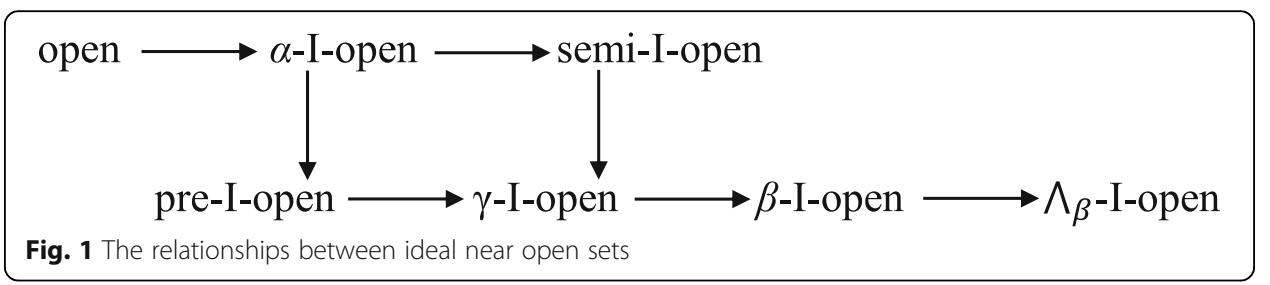


None of these implications are reversible as shown in the next example.

Example 3.2 In Example 3.1,

(i) The set $A=\{b, c, d, e\}$ is $\alpha$-I-open and it is not open.

(ii) The set $A=\{b, e\}$ is semi-I-open and it is not $\alpha-\mathrm{I}$-open.

(iii) The set $A=\{a, d, e\}$ is pre-I-open and it is not $\alpha$-I-open.

(iv) The set $A=\{a, b, e\}$ is $\gamma$-I-open and it is not pre-I-open.

(v) The set $A=\{a, b, d, e\}$ is $\gamma$-I-open and it is not semi-I-open.

(vi) The set $A=\{b, d\}$ is $\beta$-I-open and it is not $\gamma$-I-open.

(vii)The set $A=\{b\}$ is $\wedge_{\beta}$-I-open and it is not $\beta$-I-open.

\section{j-ideal approximation spaces}

In this section, we introduce and investigate the concepts of $j$-ideal lower and $j$-ideal upper approximations for any subset, where $j \in\left\{\alpha, S, P, \gamma, \beta, \wedge_{\beta}\right\}$. Some properties of these approximations will be studied and show that $\Lambda_{\beta}$-ideal is the best approximation among the others.

Definition 4.1 Let $\kappa=\left(X, R, \tau_{K}\right)$ be a topologized approximation space, $I$ be an ideal on $X, A \subseteq X$ and $\forall j \in\{\alpha, S, P, \gamma, \beta, \wedge \beta\}$. The $j$-I-lower, $j$-I-upper approximations, $j-I$ boundary, $j-I$-positive and $j-I$-negative regions, and $j$ - $I$-accuracy of the approximation of $A$ are defined respectively by:

$$
\begin{aligned}
& \underline{R}_{j I}(A)=\cup\{G \in j I O(X): G \subseteq A\}, \bar{R}_{j I}(A)=\cap\{F \in j I C(X): A \subseteq F\} \\
& B_{j I}(A)=\bar{R}_{j I}(A)-\underline{R}_{j I}(A) . \\
& \operatorname{POS}_{j I}(A)=\underline{R}_{j I}(A) . \\
& \operatorname{NEG}_{j I}(A)=X-\bar{R}_{j I}(A) . \\
& \eta_{\wedge_{\beta} I}(A)=\left|\frac{\underline{R}_{j I}(A)}{\bar{R}_{j I}(A)}\right|, \text { where }\left|\bar{R}_{j I}(A)\right| \neq 0 .
\end{aligned}
$$

Example 4.1 In Example 3.1, we construct Table 1 to show that $j I$-accuracy measure for any nonempty subset $A$ of $X$. We get the best of these methods by using $\wedge_{\beta} I$ in constructing the approximations of sets, since the boundary regions in this case are decreased (or canceled) by increasing the lower approximation and decreasing the upper approximation.

The important properties of $j$-I-lower and $j$-I-upper approximations are presented in the following proposition.

Proposition 4.1 Let $\kappa=\left(X, R, \tau_{K}\right)$ be a topologized approximation space, $I$ be an ideal on $X, A, B \subseteq X$ and $\forall j \in\{\alpha, S, P, \gamma, \beta, \wedge \beta\}$. Then, the following properties hold:

(i)

$$
\underline{R}_{j I}(A) \subseteq A \subseteq \bar{R}_{j I}(B)
$$

(ii) $\underline{R}_{j I}(\varnothing)=R_{j I}(\varnothing)=\varnothing$ and $\underline{R}_{j I}(X)=\bar{R}_{j I}(X)=X$

(iii) If $A \subseteq B$, then $\underline{R}_{j I}(A) \subseteq \underline{R}_{j I}(B)$ and $\bar{R}_{j I}(A) \subseteq \bar{R}_{j I}(B)$

(iv) 
Table $1 \mathrm{jl}$-accuracy measure for any nonempty subset $A$ of $X$

\begin{tabular}{|c|c|c|c|c|c|c|}
\hline$A \subseteq X$ & $\eta_{a /}(A)$ & $\eta_{S_{N}(A)}$ & $\eta_{P l}(A)$ & $\eta_{Y}(A)$ & $\eta_{\beta /}(A)$ & $\eta_{\Lambda_{\beta} /}(A)$ \\
\hline$\{a\}$ & 0 & 0 & 0 & 0 & 0 & 0 \\
\hline$\{b\}$ & 0 & 0 & 0 & 0 & 0 & 1 \\
\hline$\{c\}$ & 0 & 0 & 0 & 0 & 0 & 0 \\
\hline$\{d\}$ & 0 & 0 & $1 / 3$ & $1 / 2$ & $1 / 2$ & $1 / 2$ \\
\hline$\{e\}$ & $1 / 3$ & $1 / 2$ & $1 / 3$ & $1 / 2$ & $1 / 2$ & $1 / 2$ \\
\hline$\{a, b\}$ & 0 & 0 & 0 & 0 & 0 & $1 / 2$ \\
\hline$\{a, c\}$ & 0 & 0 & 0 & 0 & 0 & 0 \\
\hline$\{a, d\}$ & 0 & 0 & $1 / 4$ & $1 / 3$ & $1 / 3$ & $1 / 3$ \\
\hline$\{a, e\}$ & $2 / 3$ & 1 & $2 / 3$ & 1 & 1 & 1 \\
\hline$\{b, c\}$ & 0 & 0 & 0 & 0 & 0 & $1 / 2$ \\
\hline$\{b, d\}$ & 0 & 0 & $1 / 3$ & $1 / 3$ & $2 / 3$ & $2 / 3$ \\
\hline$\{b, e\}$ & $1 / 3$ & $2 / 3$ & $1 / 3$ & $2 / 3$ & $2 / 3$ & $2 / 3$ \\
\hline$\{c, d\}$ & $1 / 2$ & 1 & $2 / 3$ & 1 & 1 & 1 \\
\hline$\{c, e\}$ & $1 / 5$ & $1 / 5$ & $1 / 4$ & $1 / 4$ & $1 / 3$ & $1 / 3$ \\
\hline$\{d, e\}$ & $1 / 5$ & $1 / 5$ & $2 / 5$ & $2 / 5$ & $2 / 5$ & $1 / 2$ \\
\hline$\{a, b, c\}$ & 0 & 0 & 0 & 0 & 0 & $1 / 3$ \\
\hline$\{a, b, d\}$ & 0 & 0 & $1 / 4$ & $1 / 4$ & $1 / 2$ & $1 / 2$ \\
\hline$\{a, b, e\}$ & $2 / 3$ & 1 & $2 / 3$ & 1 & 1 & 1 \\
\hline$\{a, c, d\}$ & $1 / 2$ & $2 / 3$ & $1 / 2$ & $2 / 3$ & $2 / 3$ & $2 / 3$ \\
\hline$\{a, c, e\}$ & $2 / 5$ & $2 / 5$ & $1 / 2$ & $1 / 2$ & $2 / 3$ & $2 / 3$ \\
\hline$\{a, d, e\}$ & $2 / 5$ & $2 / 5$ & $3 / 5$ & $3 / 5$ & $3 / 5$ & $3 / 4$ \\
\hline$\{b, c, d\}$ & $1 / 2$ & 1 & $2 / 3$ & 1 & 1 & 1 \\
\hline$\{b, c, e\}$ & $1 / 5$ & $2 / 5$ & $1 / 4$ & $1 / 2$ & $1 / 2$ & $1 / 2$ \\
\hline$\{b, d, e\}$ & $1 / 5$ & $2 / 5$ & $3 / 5$ & $3 / 5$ & $3 / 5$ & $3 / 5$ \\
\hline$\{c, d, e\}$ & $3 / 5$ & $3 / 5$ & $3 / 5$ & $3 / 5$ & $3 / 5$ & $3 / 4$ \\
\hline$\{a, b, c, d\}$ & $1 / 2$ & $3 / 4$ & $1 / 2$ & $3 / 4$ & $3 / 4$ & $3 / 4$ \\
\hline$\{a, b, c, e\}$ & $2 / 5$ & $3 / 5$ & $1 / 2$ & $3 / 4$ & $3 / 4$ & $3 / 4$ \\
\hline$\{a, b, d, e\}$ & $2 / 5$ & $3 / 5$ & $4 / 5$ & $4 / 5$ & $4 / 5$ & $4 / 5$ \\
\hline$\{a, c, d, e\}$ & $4 / 5$ & $4 / 5$ & $4 / 5$ & $4 / 5$ & $4 / 5$ & 1 \\
\hline$\{b, c, d, e\}$ & $4 / 5$ & $4 / 5$ & $4 / 5$ & $4 / 5$ & $4 / 5$ & $4 / 5$ \\
\hline U & 1 & 1 & 1 & 1 & 1 & 1 \\
\hline
\end{tabular}

$$
\underline{R}_{j I}(A) \cup \underline{R}_{j I}(B) \subseteq \underline{R}_{j I}(A \cup B)
$$

(v)

$$
\bar{R}_{j I}(A) \cup R_{j I}(B) \subseteq R_{j I}(A \cup B)
$$

(vi)

$$
\underline{R}_{j I}(A \cap B) \subseteq \underline{R}_{j I}(A) \cap \underline{R}_{j I}(B)
$$

(vii)

$$
\bar{R}_{j I}(A \cap B) \subseteq \bar{R}_{j I}(A) \cap R_{j I}(B)
$$

(viii) 


$$
\underline{R}_{j I}\left(A^{c}\right)=\left(\bar{R}_{j I}(A)\right)^{c}
$$

(ix)

$$
\bar{R}_{j I}\left(A^{c}\right)=\left(\underline{R}_{j I}(A)\right)^{c}
$$

(x)

$$
\underline{R}_{j I}\left(\underline{R}_{j I}(A)\right)=\underline{R}_{j I}(A)
$$

(xi)

$$
\bar{R}_{j I}\left(\bar{R}_{j I}(A)\right)=\bar{R}_{j I}(A)
$$

(xii)

$$
\underline{R}_{j I}\left(\underline{R}_{j I}(A)\right) \subseteq \bar{R}_{j I}\left(\underline{R}_{j I}(A)\right)
$$

(xiii)

$$
\underline{R}_{j I}\left(\bar{R}_{j I}(A)\right) \subseteq \bar{R}_{j I}\left(\bar{R}_{j I}(A)\right)
$$

Proof: By using the properties of $j-I$-interior and $j$ - $I$-closure, the proof is obvious.

Remark 4.1 In the case of $j \in\left\{\alpha, \wedge_{\beta}\right\}$, the properties (v) and (vi) can be replaced by the following properties, respectively:

(i)

$$
\bar{R}_{j I}(A) \cup R_{j I}(B)=R_{j I}(A \cup B)
$$

(ii)

$$
\underline{R}_{j I}(A \cap B)=\underline{R}_{j I}(A) \cap \underline{R}_{j I}(B)
$$

Remark 4.2 Example 3.1 shows that the inclusion in Proposition 4.1 parts (i), (iv), (vii), (xii), and (xiii) cannot be replaced by equality relation:

(1) For part (i), if $A=\{a\}$, then $\underline{R}_{\wedge_{\beta} I}(A)=\varnothing$ and hence $A \nsubseteq \underline{R}_{\wedge_{\beta} I}(A)$. Also, if $A=\{d\}$, then $\bar{R}_{\wedge_{\beta} I}(A)=\{c, d\}$, and hence, $\bar{R}_{\wedge_{\beta} I}(A) \nsubseteq A$.

(2) For part (iv), if $A=\{a\}$ and $B=\{e\}$, then $\underline{R}_{\wedge_{\beta} I}(A)=\varnothing, \underline{R}_{\wedge_{\beta} I}(B)=\{e\}$, and $\underline{R}_{\wedge_{\beta} I}(A \cup B)$ $=\{a, e\}$.

(3) For part (vii), if $A=\{e\}$ and $B=\{a, b\}$, then $\bar{R}_{\wedge_{\beta} I}(A)=\{a, e\}, \bar{R}_{\wedge_{\beta} I}(B)=\{a, b\}$, and $\bar{R}_{\wedge_{\beta} I}(A \cap B)=\varnothing$.

(4) For part (xii), if $A=\{e\}$, then $\underline{R}_{\Lambda_{\beta} I}\left(\underline{R}_{\Lambda_{\beta} I}(A)\right)=\{e\}$ and $\bar{R}_{\wedge_{\beta} I}\left(\underline{R}_{\wedge_{\beta} I}(A)\right)=\{a, e\}$, and therefore, $\bar{R}_{\wedge_{\beta} I}\left(\underline{R}_{\wedge_{\beta} I}(A)\right) \nsubseteq \underline{R}_{\wedge_{\beta} I}\left(\underline{R}_{\wedge_{\beta} I}(A)\right)$

(5) For part (xiii), if $A=\{c\}$, then $\bar{R}_{\wedge_{\beta} I}\left(\bar{R}_{\wedge_{\beta} I}(A)\right)=\{c\}$ and $\underline{R}_{\wedge_{\beta} I}\left(\bar{R}_{\wedge_{\beta} I}(A)\right)=\varnothing$ and so $\bar{R}_{\wedge_{\beta} I}\left(\bar{R}_{\wedge_{\beta} I}(A)\right) \nsubseteq \underline{R}_{\wedge_{\beta} I}\left(R_{\wedge_{\beta} I}(A)\right)$. 
Definition 4.2 Let $\kappa=\left(X, R, \tau_{K}\right)$ be a topologized approximation space, $I$ be an ideal on $X$, and $\forall j \in\left\{\alpha, S, P, \gamma, \beta, \wedge_{\beta}\right\}$. The subset $A \subseteq X$ is called:

(i) Totally $j$-I-definable (j-I-exact), if $\underline{R}_{j I}(A)=\bar{R}_{j I}(A)=A$.

(ii) Internally $j$-I-definable, if $A=\underline{R}_{j I}(A), A \neq \bar{R}_{j I}(A)$.

(iii) Externally $j$ - $I$-definable, if $A \neq \underline{R}_{j I}(A), A=\bar{R}_{j I}(A)$.

(iv) $j$-I-undefinable (j-I-rough), if $A \neq \underline{R}_{j I}(A), A \neq \bar{R}_{j I}(A)$.

Example 4.2 In Example 3.1, the sets $\{b\},\{a, e\},\{c, d\},\{a, b, e\},\{b, c, d\}$ and $\{a, c, d, e\}$ are totally $\wedge_{\beta} I$-exact and other sets are $\wedge_{\beta} I$-rough. Also, the sets $\{d\},\{e\},\{b, d\},\{b, e\},\{d$, $e\},\{a, d, e\},\{b, d, e\},\{c, d, e\},\{a, b, d, e\}$, and $\{b, c, d, e\}$ are internally $\wedge_{\beta} I$-definable. And also, the sets $\{a\},\{c\},\{a, b\},\{a, c\},\{b, c\},\{a, b, c\},\{a, c, d\},\{a, c, e\},\{a, b, c, d\}$, and $\{a, b, c$, $e\}$ are externally $\wedge_{\beta} I$-definable.

Definition 4.3 Let $\kappa=\left(X, R, \tau_{K}\right)$ be a topologized approximation space, $I$ be an ideal on $X$, and $A, B \subseteq X$. For all $j \in\left\{\alpha, S, P, \gamma, \beta, \wedge_{\beta}\right\}, A$ is:

(i) j-I-roughly bottom included in $B\left(A_{\sim j I}^{\subseteq} B\right)$, iff $\underline{R}_{j I}(A) \subseteq \underline{R}_{j I}(B)$

(ii) $j$-I-roughly top included in $B\left(A_{\widetilde{c}_{j I}}^{\sim} B\right)$, iff $\bar{R}_{j I}(A) \subseteq \bar{R}_{j I}(B)$

(iii) $j$-I-roughly included in $\mathrm{B}\left(A \subset_{\sim}^{\sim} j I B\right)$,iff $\left(A_{\sim}^{\subseteq} B\right)$ and $\left(A \widetilde{\bar{c}}_{j I}^{\sim} B\right)$

Definition 4.4 Let $\kappa=\left(X, R, \tau_{K}\right)$ be a topologized approximation space, $I$ be an ideal on $X$, and $A, B \subseteq X$. For all $j \in\left\{\alpha, S, P, \gamma, \beta, \wedge_{\beta}\right\}, A$ is:

(i) j-I-roughly bottom equals $B\left(A_{\sim j I}^{\equiv} B\right)$, iff $\underline{R}_{j I}(A)=\underline{R}_{j I}(B)$

(ii) $j$-I-roughly top equals $B\left(A \cong_{j I} B\right)$, iff $\bar{R}_{j I}(A)=\bar{R}_{j I}(B)$

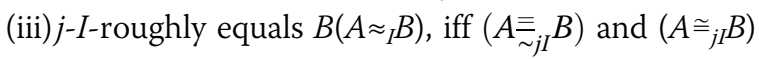

The following examples illustrate Definitions 4.3 at $j=\wedge_{\beta}$

Example 4.3 In Example 3.1, if $A=\{e\}, B=\{a, e\}, C=\{a, b\}$, and $D=\{b, e\}$, then $A$ is $\wedge_{\beta} I$-roughly bottom included in $B$ and $C$ is $\wedge_{\beta} I$-roughly top included in $D$.

The next example illustrates Definition 4.4 at $j=\wedge_{\beta}$

Example 4.4 In Example 3.1, if $A=\{d\}, B=\{a, d\}, C=\{c, e\}$, and $D=\{a, c, e\}$, then $A$ is $\wedge_{\beta} I$-roughly bottom equals $B$ and $C$ is $\wedge_{\beta} I$-roughly top equals $D$.

\section{Chemical applications}

This introduced an applied example in chemistry by applying the $j$ - $I$-approximation spaces to illustrate the concepts in a friendly way.

Example 5.1 Let $X=\left\{x_{1}, x_{2}, x_{3}, x_{4}, x_{5}\right\}$ be five amino acids (AAs). The (AAs) are described in terms of five attributes: $a_{1}=$ PIE, $a_{2}=$ SAC $=$ surface area, $a_{3}=$ MR $=$ molecular refractivity, $a_{4}=\mathrm{LAM}=$ the side-chain polarity and $a_{5}=\mathrm{Vol}=$ molecular volume [34]. Table 2 shows all quantitative attributes of five AAs.

Now, we consider five reflexive relations on $X$ defined as follow:

$$
R_{k}=\left\{\left(x_{i}, x_{j}\right) \in X \times X: x_{i}\left(a_{k}\right)-x_{j}\left(a_{k}\right)<\frac{\sigma_{k}}{2}, i, j, k=1,2, \ldots, 5\right\}
$$


Table 2 Quantitative attributes of five amino acids

\begin{tabular}{llllll}
\hline & $a_{1}$ & $a_{2}$ & $a_{3}$ & $a_{4}$ & $a_{5}$ \\
\hline$x_{1}$ & 0.23 & 254.2 & 2.126 & -0.02 & 82.2 \\
$x_{2}$ & -0.48 & 303.6 & 2.994 & -1.24 & 112.3 \\
$x_{3}$ & -0.61 & 287.9 & 2.994 & -1.08 & 103.7 \\
$x_{4}$ & 0.45 & 282.9 & 2.933 & -0.11 & 99.1 \\
$x_{5}$ & -0.11 & 335.0 & 3.458 & -0.19 & 127.5 \\
\hline
\end{tabular}

where $\sigma_{k}$ represents the standard deviation of the quantitative attributes $a_{k}, k=1,2$, $3,4,5$. The right neighborhoods for all elements of $X=\left\{x_{1}, x_{2}, x_{3}, x_{4}, x_{5}\right\}$ with respect to the relations $R_{k} k=1,2,3,4,5$ are shown in Table 3 .

We find the intersection of all right neighborhoods of all elements $k=1,2,3,4,5$ as the following:

$$
x_{1} R=\bigcap_{k=1}^{5}\left(x_{1} R_{k}\right)=\left\{x_{1}, x_{4}\right\}, x_{2} R=\bigcap_{k=1}^{5}\left(x_{2} R_{k}\right)=\left\{x_{2}, x_{5}\right\}, x_{3} R=\bigcap_{k=1}^{5}\left(x_{3} R_{k}\right)=\left\{x_{2}, x_{3},\right.
$$

$\left.x_{4}, x_{5}\right\}, x_{4} R=\bigcap_{k=1}^{5}\left(x_{4} R_{k}\right)=\left\{x_{4}\right\}$, and $x_{5} R=\bigcap_{k=1}^{5}\left(x_{5} R_{k}\right)=\left\{x_{5}\right\}$.Consider $\left\{x_{1} R, x_{2} R, x_{3} R\right.$, $\left.x_{4} R, x_{5} R\right\}$ as a base for a topology $\tau$ on $X$, then we have $\tau=\left\{X, \phi,\left\{x_{4}\right\},\left\{x_{5}\right\},\left\{x_{1}, x_{4}\right\}\right.$, $\left.\left\{x_{2}, x_{5}\right\},\left\{x_{4}, x_{5}\right\},\left\{x_{1}, x_{4}, x_{5}\right\},\left\{x_{2}, x_{4}, x_{5}\right\},\left\{x_{1}, x_{2}, x_{4}, x_{5}\right\},\left\{x_{2}, x_{3}, x_{4}, x_{5}\right\}\right\}$.

Let $I=\left\{\phi,\left\{x_{1}\right\},\left\{x_{3}\right\},\left\{x_{1}, x_{3}\right\},\left\{x_{1}, x_{4}\right\},\left\{x_{1}, x_{3}, x_{4}\right\}\right\}$ be an ideal on $X$. Then, in Table 4, the $j$-I-accuracy measure for any nonempty subset $A$ of $X$ are calculated.

In Table 4, the accuracy measures are calculated by using the current approximations in Definition 4.1. For any concept $A \subseteq X$ (collection of amino acids), this concept is determined by $\operatorname{int}_{\tau^{*}(I)}(A)$ and $\mathrm{cl}_{\tau^{*}(I)}(A)$ which defines its ideal boundary. The ideal accuracy increases by the decreases of the ideal boundary region. Clearly, the ideal accuracy measure by using the suggested class of $\wedge_{\beta} I$-open sets in general is greater than the ideal accuracy measure by using any ideal near open sets.

\section{Conclusions}

The essential goal of the rough set approach is to improve the approximation problem based on minimizing the boundary region and increase the accuracy measure. In this paper, new types of sets via ideals are investigated and some of their properties are studied. Also, the notions of $j$-ideal lower and $j$-ideal upper approximations for any subset where $j \in\left\{\alpha, S, P, \gamma, \beta, \wedge_{\beta}\right\}$ are introduced. Furthermore, some properties of these approximations are studied and show that the $\wedge_{\beta}$-ideal is the best approximation among

Table 3 Right neighborhoods of five reflexive relations

\begin{tabular}{llllll}
\hline$K$ & $x_{k} R_{1}$ & $x_{k} R_{2}$ & $x_{k} R_{3}$ & $x_{k} R_{4}$ & $x_{k} R_{5}$ \\
\hline$x_{1}$ & $\left\{x_{1}, x_{4}\right\}$ & $x$ & $x$ & $\left\{x_{1}, x_{4}, x_{5}\right\}$ & $x$ \\
$x_{2}$ & $X$ & $\left\{x_{2}, x_{5}\right\}$ & $\left\{x_{2}, x_{3}, x_{4}, x_{5}\right\}$ & $x$ & $\left\{x_{2}, x_{5}\right\}$ \\
$x_{3}$ & $X$ & $\left\{x_{2}, x_{3}, x_{4}, x_{5}\right\}$ & $\left\{x_{2}, x_{3}, x_{4}, x_{5}\right\}$ & $x$ & $\left\{x_{2}, x_{3}, x_{4}, x_{5}\right\}$ \\
$x_{4}$ & $\left\{x_{4}\right\}$ & $\left\{x_{2}, x_{3}, x_{4}, x_{5}\right\}$ & $\left\{x_{2}, x_{3}, x_{4}, x_{5}\right\}$ & $\left\{x_{1}, x_{4}, x_{5}\right\}$ & $\left\{x_{2}, x_{3}, x_{4}, x_{5}\right\}$ \\
$x_{5}$ & $\left\{x_{1}, x_{4}, x_{5}\right\}$ & $\left\{x_{5}\right\}$ & $\left\{x_{5}\right\}$ & $\left\{x_{1}, x_{4}, x_{5}\right\}$ & $\left\{x_{5}\right\}$ \\
\hline
\end{tabular}


Table 4 Comparison of the $j$-l-accuracy measures

\begin{tabular}{|c|c|c|c|c|c|c|}
\hline$A \subseteq X$ & $\eta_{a \prime}(A)$ & $\eta_{S I}(A)$ & $\eta_{P_{1}}(A)$ & $\eta_{Y^{\prime}}(A)$ & $\eta_{\beta /}(A)$ & $\eta_{\Lambda_{\beta} /}(A)$ \\
\hline$\left\{x_{1}\right\}$ & 0 & 0 & 0 & 0 & 0 & 0 \\
\hline$\left\{x_{2}\right\}$ & 0 & 0 & 0 & 0 & 0 & 0 \\
\hline$\left\{x_{3}\right\}$ & 0 & 0 & 0 & 0 & 0 & 1 \\
\hline$\left\{x_{4}\right\}$ & $1 / 3$ & $1 / 2$ & $1 / 3$ & $1 / 2$ & $1 / 2$ & $1 / 2$ \\
\hline$\left\{x_{5}\right\}$ & $1 / 3$ & $1 / 2$ & $1 / 3$ & $1 / 2$ & $1 / 2$ & $1 / 2$ \\
\hline$\left\{x_{1}, x_{2}\right\}$ & 0 & 0 & 0 & 0 & 0 & 0 \\
\hline$\left\{x_{1}, x_{3}\right\}$ & 0 & 0 & 0 & 0 & 0 & $1 / 2$ \\
\hline$\left\{x_{1}, x_{4}\right\}$ & $2 / 3$ & 1 & $2 / 3$ & 1 & 1 & 1 \\
\hline$\left\{x_{1}, x_{5}\right\}$ & $1 / 4$ & $1 / 3$ & $1 / 4$ & $1 / 3$ & $1 / 3$ & $1 / 3$ \\
\hline$\left\{x_{2}, x_{3}\right\}$ & 0 & 0 & 0 & 0 & 0 & $1 / 2$ \\
\hline$\left\{x_{2}, x_{4}\right\}$ & $1 / 4$ & $1 / 3$ & $1 / 4$ & $1 / 3$ & $1 / 3$ & $1 / 3$ \\
\hline$\left\{x_{2}, x_{5}\right\}$ & $2 / 3$ & 1 & $2 / 3$ & 1 & 1 & 1 \\
\hline$\left\{x_{3}, x_{4}\right\}$ & $1 / 3$ & $2 / 3$ & $1 / 3$ & $2 / 3$ & $2 / 3$ & $2 / 3$ \\
\hline$\left\{x_{3}, x_{5}\right\}$ & $1 / 3$ & $2 / 3$ & $1 / 3$ & $2 / 3$ & $2 / 3$ & $2 / 3$ \\
\hline$\left\{x_{4}, x_{5}\right\}$ & $2 / 5$ & $2 / 5$ & $2 / 5$ & $2 / 5$ & $2 / 5$ & $1 / 2$ \\
\hline$\left\{x_{1}, x_{2}, x_{3}\right\}$ & 0 & 0 & 0 & 0 & 0 & $1 / 3$ \\
\hline$\left\{x_{1}, x_{2}, x_{4}\right\}$ & $1 / 2$ & $2 / 3$ & $1 / 2$ & $2 / 3$ & $2 / 3$ & $2 / 3$ \\
\hline$\left\{x_{1}, x_{2}, x_{5}\right\}$ & $1 / 2$ & $2 / 3$ & $1 / 2$ & $2 / 3$ & $2 / 3$ & $2 / 3$ \\
\hline$\left\{x_{1}, x_{3}, x_{4}\right\}$ & $2 / 3$ & 1 & $2 / 3$ & 1 & 1 & 1 \\
\hline$\left\{x_{1}, x_{3}, x_{5}\right\}$ & $1 / 4$ & $1 / 2$ & $1 / 4$ & $1 / 2$ & $1 / 2$ & $1 / 2$ \\
\hline$\left\{x_{1}, x_{4}, x_{5}\right\}$ & $3 / 5$ & $3 / 5$ & $3 / 5$ & $3 / 5$ & $3 / 5$ & $3 / 4$ \\
\hline$\left\{x_{2}, x_{3}, x_{4}\right\}$ & $1 / 4$ & $1 / 2$ & $1 / 4$ & $1 / 2$ & $1 / 2$ & $1 / 2$ \\
\hline$\left\{x_{2}, x_{3}, x_{5}\right\}$ & $2 / 3$ & 1 & $2 / 3$ & 1 & 1 & 1 \\
\hline$\left\{x_{2}, x_{4}, x_{5}\right\}$ & $3 / 5$ & $3 / 5$ & $3 / 5$ & $3 / 5$ & $3 / 5$ & $3 / 4$ \\
\hline$\left\{x_{3}, x_{4}, x_{5}\right\}$ & $3 / 5$ & $3 / 5$ & $3 / 5$ & $3 / 5$ & $3 / 5$ & $3 / 5$ \\
\hline$\left\{x_{1}, x_{2}, x_{3}, x_{4}\right\}$ & $1 / 2$ & $3 / 4$ & $1 / 2$ & $3 / 4$ & $3 / 4$ & $3 / 4$ \\
\hline$\left\{x_{1}, x_{2}, x_{3}, x_{5}\right\}$ & $1 / 2$ & $3 / 4$ & $1 / 2$ & $3 / 4$ & $3 / 4$ & $3 / 4$ \\
\hline$\left\{x_{1}, x_{2}, x_{4}, x_{5}\right\}$ & $4 / 5$ & $4 / 5$ & $4 / 5$ & $4 / 5$ & $4 / 5$ & 1 \\
\hline$\left\{x_{1}, x_{3}, x_{4}, x_{5}\right\}$ & $4 / 5$ & $4 / 5$ & $4 / 5$ & $4 / 5$ & $4 / 5$ & $4 / 5$ \\
\hline$\left\{x_{2}, x_{3}, x_{4}, x_{5}\right\}$ & $4 / 5$ & $4 / 5$ & $4 / 5$ & $4 / 5$ & $4 / 5$ & $4 / 5$ \\
\hline U & 1 & 1 & 1 & 1 & 1 & 1 \\
\hline
\end{tabular}

the others. Finally, a chemical application which may give a solution for real-life problems will be discussed.

\section{Future work}

The following points will be studied in the future:

1. We define the notion $\theta \beta$-open sets in ideal topological spaces as a generalization of $j$-ideal topological spaces where $j \in\{\alpha, S, P, \gamma, \beta, \quad \beta\}$.

2. We introduce and investigate the concepts of $\theta \beta$-ideal lower and $\theta \beta$-ideal upper approximations for any subset.

3. New applications of these new approximations in various real-life fields. 
Acknowledgements

The author would like to thank the editors for a careful and thorough reading of this manuscript.

\section{Authors' contributions}

A.S.N is the main and only author of this paper. The author read and approved the final manuscript.

Funding

There is no funding available.

Availability of data and materials

Not applicable

Competing interests

The author declares that there are no competing interests.

Received: 5 August 2019 Accepted: 30 December 2019

Published online: 14 January 2020

\section{References}

1. Pawlak, Z:: Rough sets. International Journal of Computer and Information Sciences. 11, 341-356 (1982)

2. Pawlak, Z:: Rough sets: theoretical aspects of reasoning about data. Kluwer Academic Publishers, Boston (1991)

3. Chmielewshi, M.R., Grzymala-Busse, J.W.: Global discretization of continuous attributes as preprocessing for machine learning. Int. J. Approx. Reason. 15(4), 319-331 (1966)

4. Ziarko, W.: The discovery, analysis, and representation of data dependencies in databases. In: Piatetsky-Shapiro, G. Frawley, W.G. (eds.) Knowledge Discovery in Databases, pp. 213-228. MIT Press, Cambridge, MA (1990)

5. Chan, C.C.: A rough set approach to attribute generalization in data mining. J. Inf. Sci. 107, 169-176 (1998)

6. Lingras, P.J., Yao, Y.Y.: Data mining using extensions of the rough set model. J. Amer. Soc. Inf. Sci. 49(5), 415-422 (1998)

7. Mcsherry, D.: Knowledge discovery by inspection. Decis. Support Syst. 21(1), 43-47 (1997)

8. Pawlak, Z:: Rough set approach to knowledge-based decision support. Eur. J. Oper. Res. 99(1), 48-57 (1997)

9. Pomerol, J.C.: Artificial intelligence and human decision making. Eur. J. Oper. Res. 99(1), 3-25 (1997)

10. Pawlak, Z., et al.: Rough sets. Commun. ACM. 38(11), 89-95 (1995)

11. Peters, J.F., Ziaei, K., Ramanna, S.: Approximate time rough control: concepts and application to satellite attitude control, in Proc. RSCTC. 491-498 (1998)

12. Yahia, M.E., Mahaod, R., Sulaiman, N., Ahamad, F.: Rough neural expert systems. Expert Syst. 18(2), 87-99 (2000)

13. Meng-Xin, L., Cheng-Dong, W., Xing-Hua, X., Yue, Y.: Rough set theory and its application. J. Shenyang Archit. Civil Eng. Univ. 17, 296-299 (2001)

14. Kuratowski, K.: Topology, vol. 1. Academic press, New York (1966)

15. Vaidyanathaswamy, R.: The localisation theory in set topology. Proc. Indian Acad. Sci. 20, 51-61 (1945)

16. D. S.Jankovic and T.R.Hamlett, New topologies from old via ideals, Amer. Math. Monthly, 97 (4)(1990), 295-310.

17. Mashhour, A.S., Abd El-Monsef, M.E., El-Deeb, S.N.: On precontinuous and weak precontinuous mappings. Proc. Math. Phys. Soc. Egypt. 53, 47-53 (1982)

18. Levine, N.: Semi-open sets and semi-continuity in topological spaces. Amer. Math. Monthly. 70, 36-41 (1963)

19. Andrijevic, D.: On b-open sets. Mat. Vesnik. 48, 59-64 (1996)

20. A.A. El-Atik, A study of some types of mappings on topological spaces, M.Sc. Thesis, Tanta Univ. Egypt, 1997.

21. Andrijevic, D.: Semi-preopen sets. Mat. Vesnik. 38(1), 24-32 (1986)

22. M E Abd EL-Monsef, S. N. El-Deeb and R. A. Mahmoud, $\beta$-open sets and $\beta$-continuous mappings, Bull. Fac. Sci. Assiut Univ., 12 (1983), 77-90.

23. Njåstad, O.: On some classes of nearly open sets. Pacific. J. Math. 15, 961-970 (1965)

24. Noiri, T., Hatir, E.: $\wedge_{s p}$-sets and some weak separation axioms. Acta Mathematica Hungarica. 103(3), 225-232 (2004)

25. J.Dontchev, On pre---open sets and a decomposition of I-continuity, Banyan Math. J., 2 (1996).

26. Hatir, E., Noiri, T.: On decompositions of continuity via idealization. Acta Math. Hungar. 96(4), $341-349$ (2002)

27. A. Caksu Guler and G. Aslim, b-l-open sets and decomposition of continuity via idealization, Proceedings of Institute of Mathematics and Mechanics. National Acedemy of Sciences of Azerbaijan, 22(2005), 27-32.

28. T.Y. Lin, Topological and fuzzy rough sets, in: R. Slowinski (Ed.), Decision Support by Experience-Application of the Rough Sets Theory, Kluwer Academic Publishers, (1992), 287-304.

29. Pawlak, Z:: Rough sets: theoretical aspects of reasoning about data, vol. 9. Kluwer Academic Publishers, Dordrecht (1991)

30. Pawlak, Z., Skowron, A.: Rough sets: some extensions. Information Sciences. 177, 28-40 (2007)

31. Abd El-Monsef, M.E., Kozae, A.M., Iqelan, M.J.: Near approximations in topological spaces. Int. Journal of Math. Analysis. 4(6), 279-290 (2010)

32. Lashin, E., Kozae, A., Abo Khadra, A., Medhat, T.: Rough set theory for topological spaces. International Journal of Approximate Reasoning. 40, 35-43 (2005)

33. Abu-Donia, H.M.: New rough set approximation spaces. Abstract and Applied Analysis. 1-7 (2013)

34. Abu-Donia, H.M., Salama, A.S.: Generalization of Pawlaks rough approximation spaces by using $\delta \beta$-open sets. International Journal of Approximate Reasoning. 53(7), 1094-1105 (2012)

\section{Publisher's Note}

Springer Nature remains neutral with regard to jurisdictional claims in published maps and institutional affiliations. 